\title{
HERMES: a high-resolution fibre-fed spectrograph for the Mercator telescope ${ }^{\star}$
}

\author{
G. Raskin ${ }^{1}$, H. Van Winckel ${ }^{1}$, H. Hensberge ${ }^{2}$, A. Jorissen ${ }^{3}$, H. Lehmann ${ }^{4}$, C. Waelkens ${ }^{1}$, G. Avila ${ }^{5}$, J.-P. De Cuyper ${ }^{2}$, \\ P. Degroote ${ }^{1}$, R. Dubosson ${ }^{6}$, L. Dumortier ${ }^{2}$, Y. Frémat ${ }^{2}$, U. Laux $^{4}$, B. Michaud ${ }^{6}$, J. Morren ${ }^{7}$, J. Perez Padilla ${ }^{1}$, \\ W. Pessemier ${ }^{1}$, S. Prins ${ }^{1}$, K. Smolders ${ }^{1}$, S. Van Eck $^{3}$, and J. Winkler ${ }^{4}$

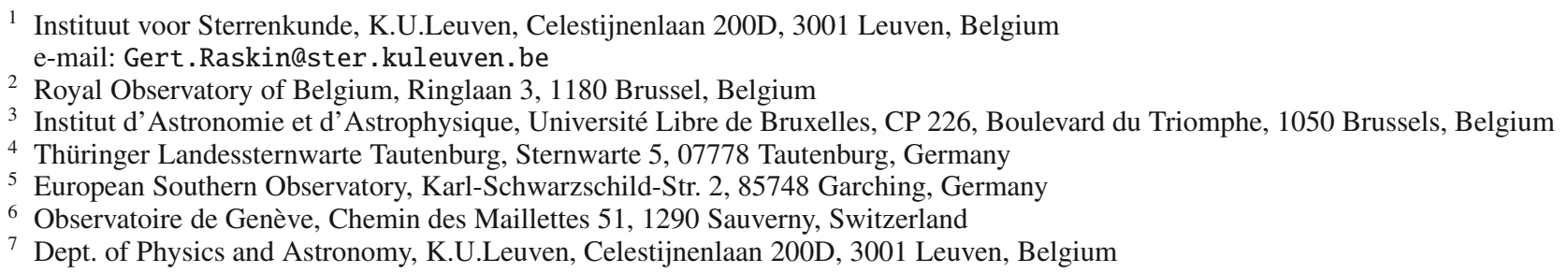

Received 19 July 2010 / Accepted 11 October 2010

\begin{abstract}
The HERMES high-resolution spectrograph project aims at exploiting the specific potential of small but flexible telescopes in observational astrophysics. The optimised optical design of the spectrograph is based on the well-proven concept of white-pupil beam folding for high-resolution spectroscopy. In this contribution we present the complete project, including the spectrograph design and procurement details, the telescope adaptor and calibration unit, the detector system, as well as the optimised data-reduction pipeline. We present a detailed performance analysis to show that the spectrograph performs as specified both in optical quality and in total efficiency. With a spectral resolution of 85000 (63000 for the low-resolution fibre), a spectral coverage from 377 to $900 \mathrm{~nm}$ in a single exposure and a peak efficiency of $28 \%$, HERMES proves to be an ideal instrument for building up time series of high-quality data of variable (stellar) phenomena.
\end{abstract}

Key words. instrumentation: spectrographs - techniques: spectroscopic - techniques: radial velocities - stars: abundances

\section{Introduction}

The Mercator telescope (www.mercator.iac.es) is a $1.2-\mathrm{m}$ optical telescope based at the Roque de los Muchachos Observatory on La Palma (Canary Islands, Spain) and funded by the Flemish community of Belgium. The telescope is operated by the Instituut voor Sterrenkunde of the K.U. Leuven university (Belgium). The telescope itself is a twin telescope of the Swiss Euler Telescope operational at the La Silla observatory of the European Southern Observatory (ESO). Both telescopes are ideal complements to international facilities. Their specific niche in observational astrophysics is that they guarantee long and secured access, which allows for dedicated monitoring programmes of variable phenomena.

To exploit the telescope infrastructure, a focused instrument development programme was deployed and in this contribution we present HERMES, an acronym for high efficiency and resolution Mercator echelle spectrograph. This instrument was realised by a consortium of institutes with main science drivers in stellar astrophysics. The project was led by the Instituut voor Sterrenkunde (K.U. Leuven, Belgium) with as main partners the Institut d'Astronomie et Astrophysique (ULB, Belgium) and the Royal Observatory of Belgium, and with smaller contributions

^ Based on observations made with the Mercator Telescope, operated on the island of La Palma by the Flemish Community, at the Spanish Observatorio del Roque de los Muchachos of the Instituto de Astrofísica de Canarias. from the Thüringer Landessternwarte Tautenburg (Germany) and the Geneva Observatory (Switzerland).

The science drivers cover a wide range of topics in stellar astrophysics that mainly call for dedicated high-quality time series of high-resolution spectra. They comprise the ground-based follow-up of asteroseismology space missions and spectroscopic asteroseismology studies in general (e.g. Aerts et al. 2010), massive (interacting) binaries (e.g. Hensberge et al. 2008; Lehmann et al. 2010; Blomme 2010), final phases of stellar evolution in (binary) stars (e.g. Van Winckel et al. 2009; Jorissen et al. 2009), and photospheric abundance determinations as probes to internal nucleosynthesis (e.g. Masseron et al. 2010; Reyniers et al. 2007). This resulted in a series of science requirements that cover spectrograph characteristics (spectral resolution $>80000$; spectral range $>380-880 \mathrm{~nm}$; throughput $>25 \%$ in $V$, cycle time $<60 \mathrm{~s}$; radial velocity stability $<5 \mathrm{~m} \mathrm{~s}^{-1}$ ), as well as operational goals (flexible but robust night operations). The last are important, given the limited local staff and night support at the Mercator telescope. To accommodate these requirements, a fibre-fed spectrograph with two science modes was designed: one optimised for high spectral resolution with good wavelength stability and excellent throughput, and the other optimised for very high stability with lower resolution and throughput.

In this contribution we present the HERMES spectrograph project, which was realised in between the kick-off meeting of 19 January 2005 and the start of the regular science operations in April 2009. We start with a description of the instrument design 


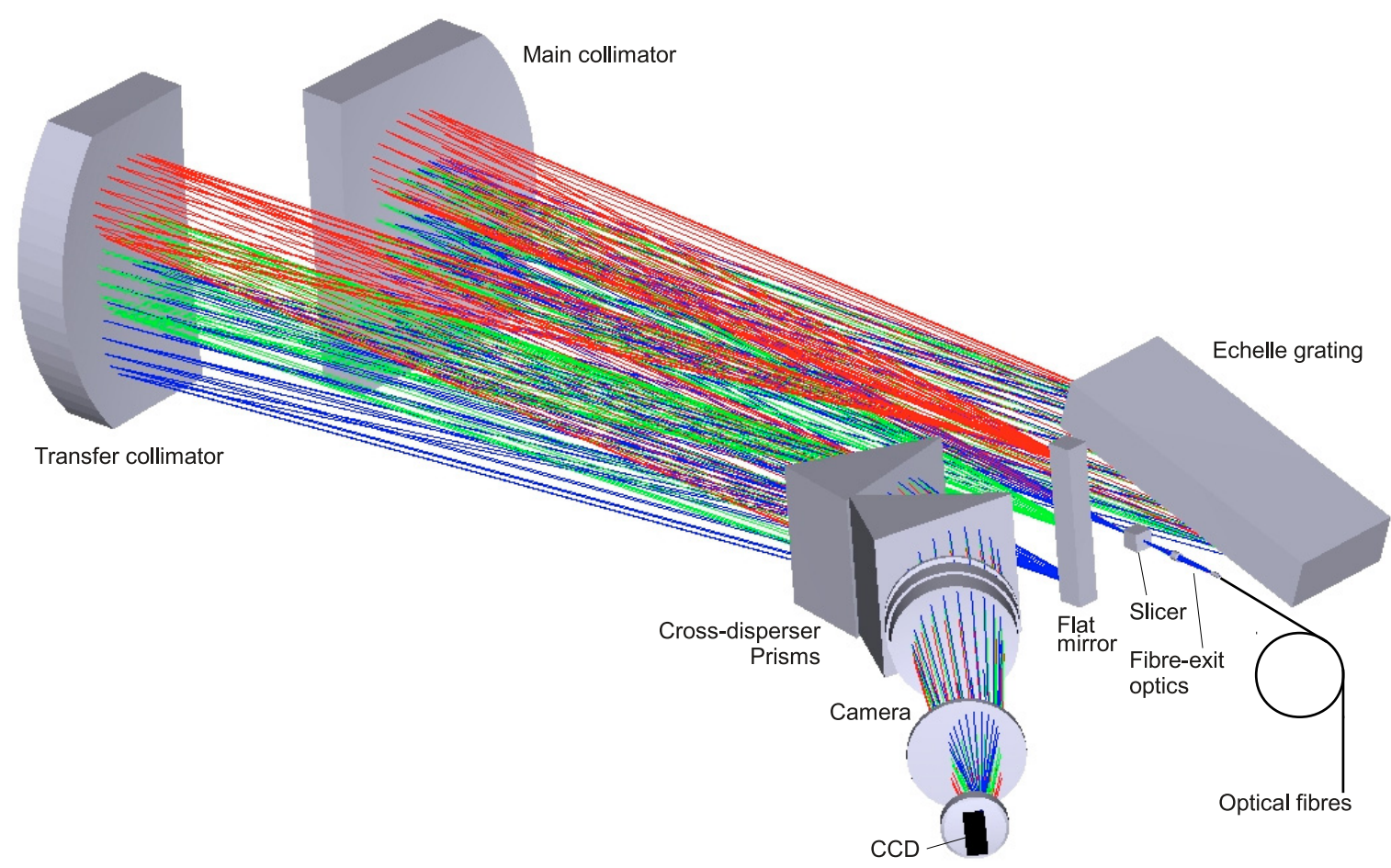

Fig. 1. 3D view of the HERMES optical layout.

in Sect. 2 and of the data-reduction pipeline in Sect. 3. In Sect. 4 we report on the performance analyses, followed by conclusions in Sect. 5.

\section{Instrument design}

\subsection{Spectrograph}

\subsubsection{Optical design}

Like many present-day high-resolution spectrographs, the optical layout of HERMES (Fig. 1) is based on a white-pupil design, discussed in detail by Baranne (1988). This concept implemented with a pair of off-axis parabolas as collimator, as first done for UVES (Dekker et al. 2000), makes it arguably one of the best solutions for a high-resolution spectrograph. It was also used for other successful ESO spectrographs like FEROS (Kaufer \& Pasquini 1998), generally accepted as a benchmark for instrument efficiency, and HARPS (Pepe et al. 2000) as a benchmark for radial-velocity precision. These projects became important inspirations for HERMES, which attempted to combine the strengths of these instruments. These instruments are also fibre-fed, and the HERMES design follows their wellproven concept.

Echelle grating. HERMES uses an echelle grating blazed with an intermediate blaze angle of $\theta=69.74^{\circ}(\tan \theta=2.7)$ as main dispersing element. The ruling of 52.676 grooves $/ \mathrm{mm}$ nicely distributes the spectrum from $377 \mathrm{~nm}$ to $900 \mathrm{~nm}$ in 55 spectral orders over the rectangular CCD. The aluminium-coated ruled surface on a Zerodur blank measures $154 \times 408 \mathrm{~mm}$. The grating is used in Littrow condition, but is slightly tilted $\left(\gamma=0.8^{\circ}\right)$ perpendicular to the direction of dispersion to separate the dispersed from the incident beam. To avoid dust contamination, the grating is mounted with the optical surface facing downwards. The efficiency of the grating at blaze peak amounts to $65 \%$.

Collimators. The HERMES collimators were cut from a single 660-mm diameter Zerodur parabolic mirror with a focal length of $1400 \mathrm{~mm}$ (Seso, France). With an aperture of $f / 9.2$, the collimated beam has a diameter of $152 \mathrm{~mm}$. The mirrors are coated with a blue-enhanced protected silver coating (Spectrum Thin Films, USA) with very good reflectivity in the blue and green parts of the spectrum (Refl. > 96\% at $400 \mathrm{~nm}$, Refl. $>99 \%$ over $450-650 \mathrm{~nm}$ ) but with a somewhat disappointing and below-specification performance in the near-infrared (Refl. $>94 \%$ at $800 \mathrm{~nm}$ ).

Cross disperser. Two identical prisms used at minimum deviation for the spectrograph's central wavelength $(490 \mathrm{~nm})$ separate the spatially overlapping echelle orders. Thanks to the whitepupil mounting, the size of the cross-disperser prisms could be minimised, but they still are two impressive blocks of glass with a base of $138 \times 208 \mathrm{~mm}$, a height of $197 \mathrm{~mm}$, and an apex angle of $37.4^{\circ}$. Both prisms are made of Ohara I-Line PBL1Y glass with very high blue and UV transmission (Optique Fichou, France). The cross disperser provides a minimum order separation of 24 pixels in the reddest part of the spectrum, a value that increases to 55 pixels at $380 \mathrm{~nm}$. The inter-order distance is sufficiently large to record two interleaved spectra in case of the simultaneous thorium technique (Baranne et al. 1996), separated by 13 pixels on the CCD.

The ratio between the longest and shortest wavelength is 2.4 for HERMES. The rejection of unwanted orders over such a broad spectral range is complicated when using a gratingtype cross disperser. Moreover, the prism solution has higher 

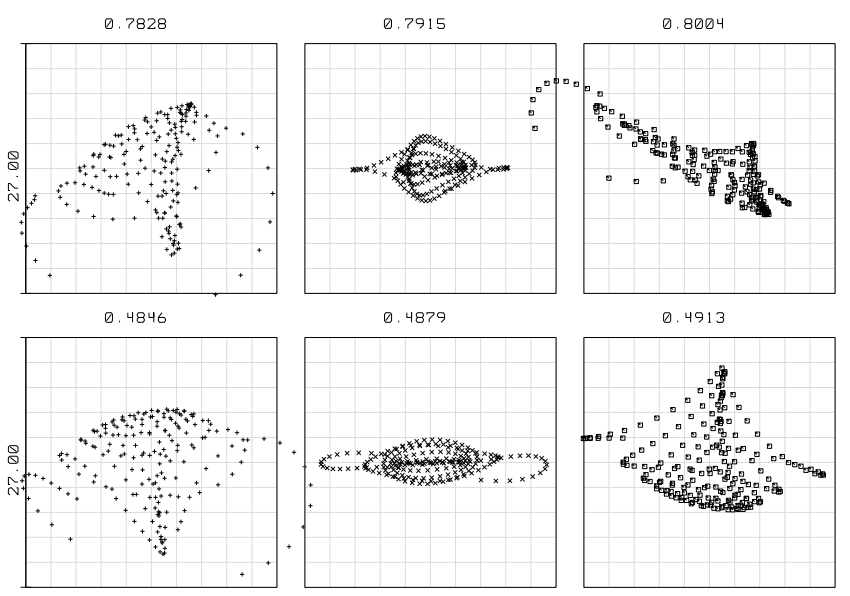

0.3830
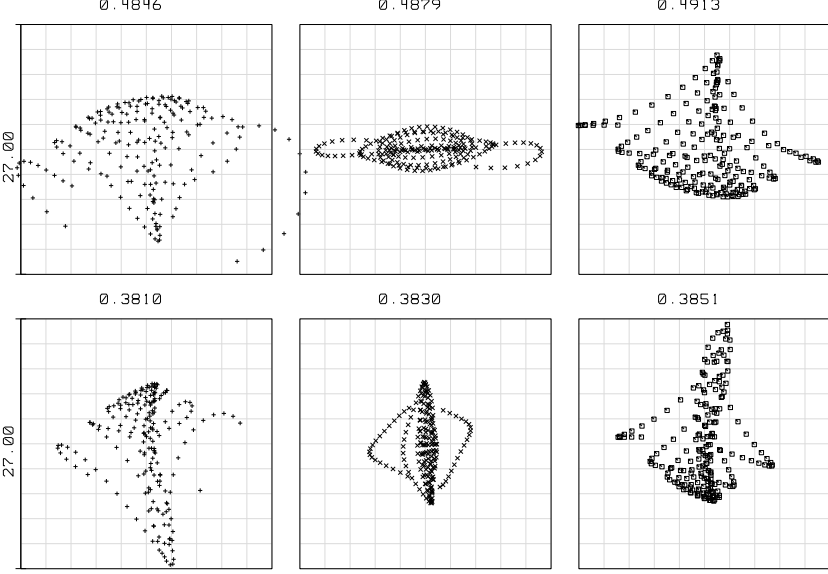

0.3851

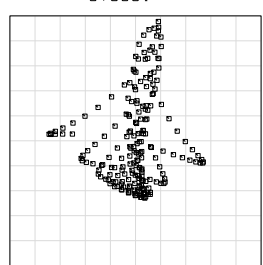

Fig. 2. HERMES spot diagrams at beginning, centre, and end of the free spectral range for three representative orders (43, 73, and 93). Wavelength is indicated in $\mu \mathrm{m}$ above each $2 \times 2$ pixels box $(27 \mu \mathrm{m})$.

efficiency (20-30\%), avoids the production of cross-order scattered light, and offers more evenly spaced orders. We therefore discarded the use of a grating as in UVES (Dekker et al. 2000) or a grism as in HARPS (Pepe et al. 2000).

Dioptric camera. The camera (Seso, France) that focuses the spectrum on the detector is a fully dioptric system consisting of three singlet lenses and one large cemented triplet. The last lens acts as field flattener with a convex cylindrical rear surface. This lens also serves as a vacuum window of the detector cryostat. The camera has a focal length of $475 \mathrm{~mm}$ and a focal ratio of $f / 3.1$. This gives the spectrograph a linear dispersion of $0.002 \mathrm{~nm} / \mathrm{pixel}$ at the centre of the bluest order, increasing to $0.0047 \mathrm{~nm} /$ pixel for the reddest order. The camera characteristics are calculated to image all but the reddest spectral orders without gaps on the detector. From $838 \mathrm{~nm}$ onwards, 3 orders are slightly truncated. Sacrificing lateral colour correction completely, the moderate complexity of this camera nonetheless delivers excellent image quality as can be appreciated from the spot diagrams for the complete optical system of the spectrograph in Fig. 2. The overall throughput of the camera is higher than $88 \%$ for most of the wavelength range.

Fibre-exit optics and exposure metre. The light beam exiting the optical fibres enters the spectrograph at a $f / 3.7$ focal ratio. This is converted to a $f / 9.2$ beam - accepted by the collimators - by a set of focal-ratio-adaptation optics, consisting of one doublet and one triplet (Precision Optics Gera, Germany). The doublet is cemented directly onto the fibres for increased mechanical stability and to limit the number of glass-air interfaces, thereby reducing reflection losses.

In between the two lenses and conjugated with the echelle grating, the inclined reflective stop of the adaptation optics collects those photons otherwise lost from the main beam due to focal ratio degradation (see Sect. 2.2) and sends them to a

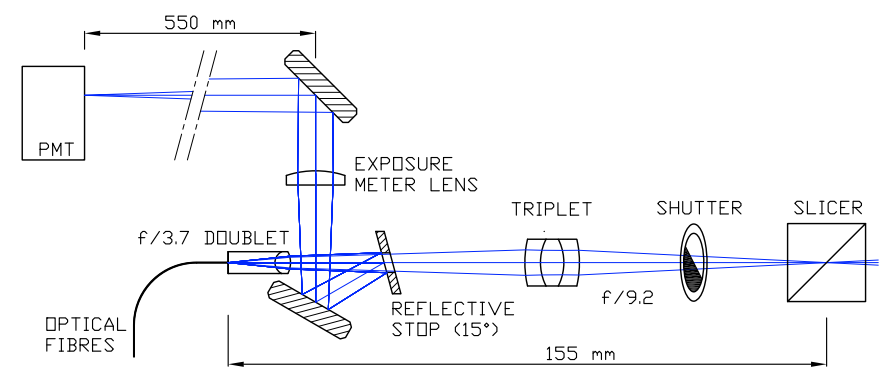

Fig. 3. Optical fibres entering the spectrograph, focal-ratio-adaptation optics, exposure metre and slicer.

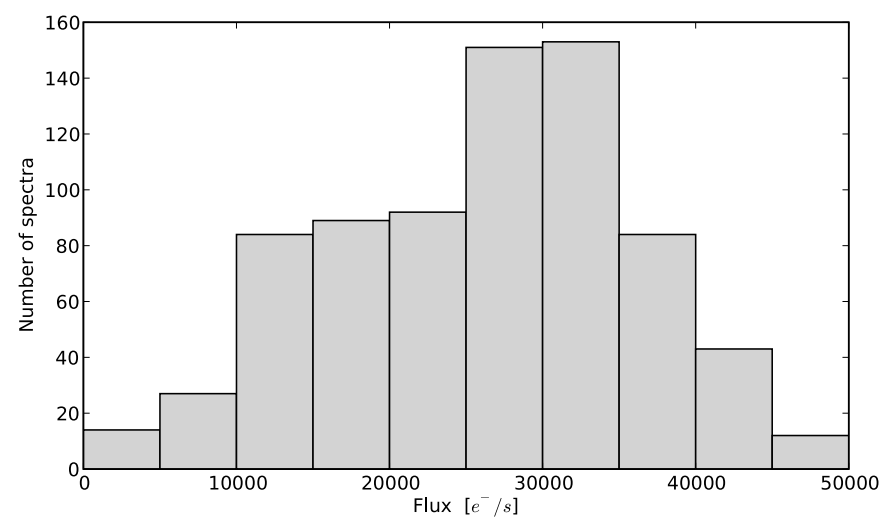

Fig. 4. Histogram of the flux measured in V, normalised to the 0th magnitude, for a large sample of HERMES spectra.

photo-multiplier tube for continuous flux monitoring. One lens and two fold mirrors image the fibres on the photo-multiplier detector (Fig. 3). The flux from the thorium fibre is masked out to avoid contamination of the stellar flux. This exposure metre allows the determination of accurate photon-weighted mean time of the exposures, necessary to correct for the Earth's velocity. It is also very useful for matching the exposure time with the desired signal-to-noise ratio $(S / N)$ of the final spectrum during observations under poor weather conditions. The broad distribution of the flux histogram in Fig. 4 clearly illustrates the effect of variable observing conditions and the resulting need for continuous flux monitoring.

Slicer. A two-slice image slicer effectively doubles the spectral resolution in the high-resolution mode. As the cross-order profile of the sliced fibre is too wide or the inter-order spacing too small to sample two interleaved and sliced spectra, the slicer cannot be used for simultaneous thorium exposures. For the same reason a simultaneous sky fibre for sky substraction was not implemented. The image slicer is similar to the FEROS type (Kaufer 1998) but here only one fibre image is sliced. The images of the two fibres used in the simultaneous thorium mode pass unsliced through the slicer. The slicer layout and operation are shown in Fig. 5.

The design of the slicer allows imaging of the sliced and unsliced fibres without any focus difference. Only a negligible focus difference of $0.25 \mathrm{~mm}$ exists between the two slices of the sliced image. The slicer consists of an entrance prism with one air groove, the slicer plate whose thickness $(0.173 \mathrm{~mm})$ determines the distance between the two slices $(0.277 \mathrm{~mm})$, and the cut-off slicer prism. The prism angle is $46.5^{\circ}$ guaranteeing total internal reflection up to the longest wavelengths. The sides of the prism measure $25 \mathrm{~mm}$. All parts are made from Homosil quartz 


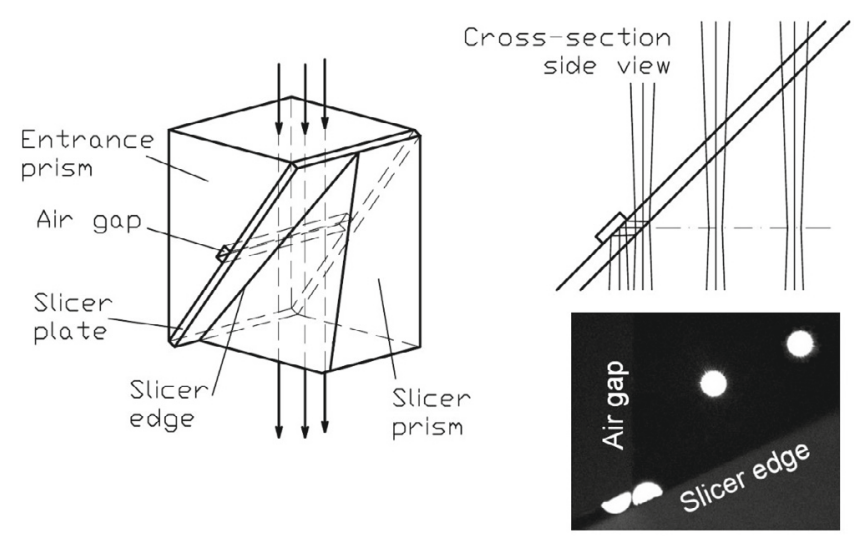

Fig. 5. Perspective view and cross-section of the slicer. The picture shows an image of the simultaneously illuminated sliced and unsliced fibres. Inside the spectrograph, the slicer edge is aligned horizontally.

and connected by optical contact (Horst Kaufmann, Germany). The entrance and exit surfaces of the slicer are coated with a broadband AR coating. The very high throughput of the slicer (above 95\%) largely compensates for the increased complexity compared to the use of a slit for obtaining higher resolution.

\subsubsection{Mechanical design}

Mechanical stability is a main issue in the design of a spectrograph for measuring precise radial velocities. HERMES is therefore mounted on a vibration-absorbing optical bench $(2400 \times 1200 \times 300 \mathrm{~mm})$. For more mechanical stability, this bench is located on a solid concrete foundation, isolated from the rest of the telescope building. As the spectrograph works in a completely fixed configuration, there are no moving parts on the optical bench that might compromise this stability with the exception of a tiny shutter, installed at the fibre exit where the beam diameter is minimal (Fig. 3). All large optical elements have (semi-)kinematic mountings that constrain all six degrees of freedom independently and without redundancy.

Although the temperature of the spectrograph room is precisely controlled (see Sect. 2.1.3), special care was taken with the thermal stability of the opto-mechanics as well. Most of the mounts for the optical elements are manufactured from cast iron because of its excellent resistance to deformation and, more importantly, its coefficient of thermal expansion (CTE), which matches the CTE of the ferromagnetic stainless steel of the spectrograph bench quite well. Moreover, the mounts of the most critical optics incorporate some type of passive thermal stabilisation to reduce the sensitivity to temperature variations:

- Thermal expansion of both the metal structures of the spectrograph and the lenses (mostly in the camera), as well as thermal changes in the refractive index of air and lens glasses, have a non-negligible effect on the spectrograph focus. Temperature changes greater than $1{ }^{\circ} \mathrm{C}$ would require a focus correction or would result in resolution loss. The focusing mechanism of the camera is therefore equipped with a thermal compensation system that maintains the spectrograph in focus over a broad temperature range. Figure 6 illustrates the insensitivity of the spectrograph focus to temperature variations of $\pm 3{ }^{\circ} \mathrm{C}$, together with the expected defocus without compensation calculated by ZEMAX. Thermal compensation is based on a 520-mm long Invar rod that pushes against the camera barrel through a lever. The opposite side

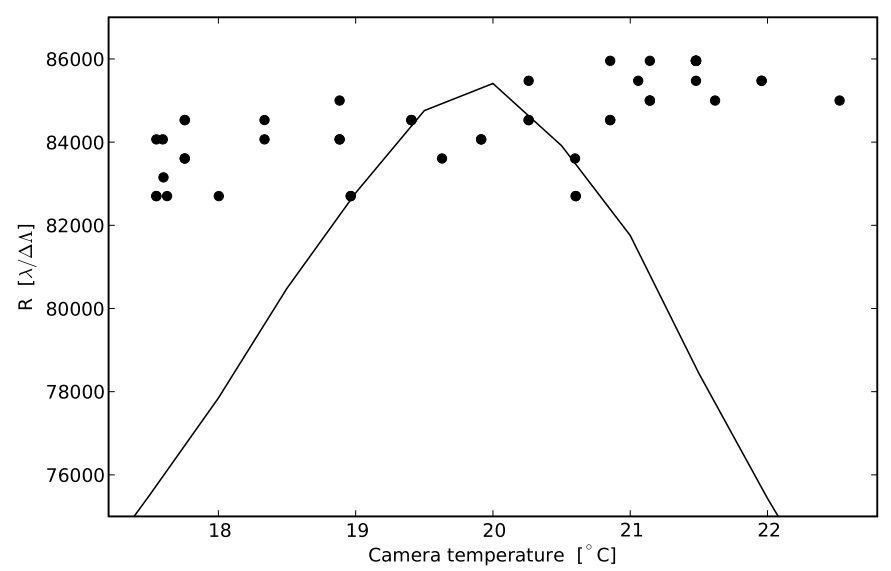

Fig. 6. Effect of temperature on spectral resolution or spectrograph focus (dots). The theoretical thermal sensitivity based on a ZEMAX model without compensation is shown as a solid line for comparison.

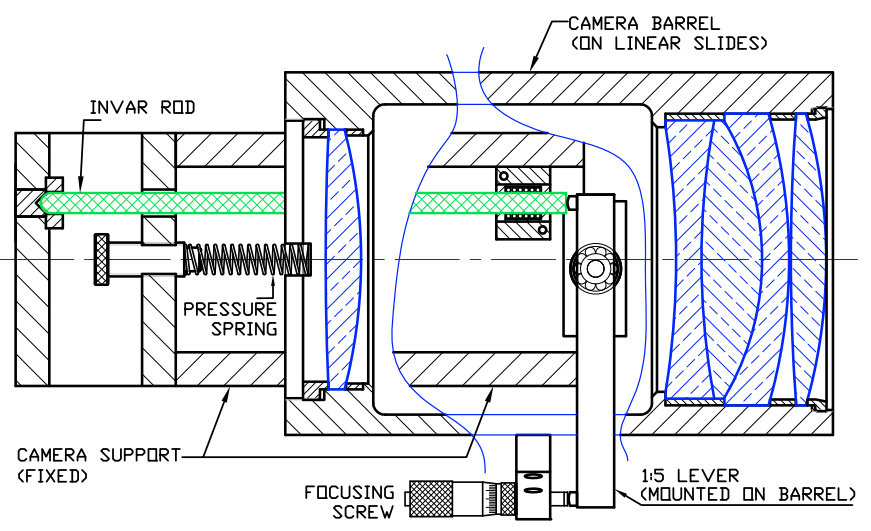

Fig. 7. Camera focusing mechanism with temperature compensation.

of the lever holds the focusing screw (Fig. 7). Differential thermal expansion of the Invar rod and the cast iron camera support, amplified by the 1:5 lever, keep the spectrograph well-focused independently of temperature.

- The cryostat support and the detector support inside the cryostat are designed to have their centre of thermal expansion exactly on the optical axis of the spectrograph camera.

- The vertical position of the fibre-exit unit is precisely kept at the focal point of the Zerodur parabolas through a tuned combination of Invar and brass in the fibre support.

- Similarly, the angular position of the grating is thermally neutralised by the differential thermal expansion of Invar and stainless steel in the kinematic grating supports. Figure 8 shows the fourfold reduction in the thermal sensitivity when using thermally compensated kinematic supports. Imperfect modeling left us with a drift of 0.22 pixels $/{ }^{\circ} \mathrm{C}$, but this can be further reduced empirically.

\subsubsection{Environment control}

Precise control of the spectrograph environment is critical for the long-term stability of the instrument, with temperature and air pressure the most important parameters. Fluctuations in the relative humidity $(\mathrm{RH})$ are less relevant as they have less influence on the refractive index of air. In any case, the air conditioning 


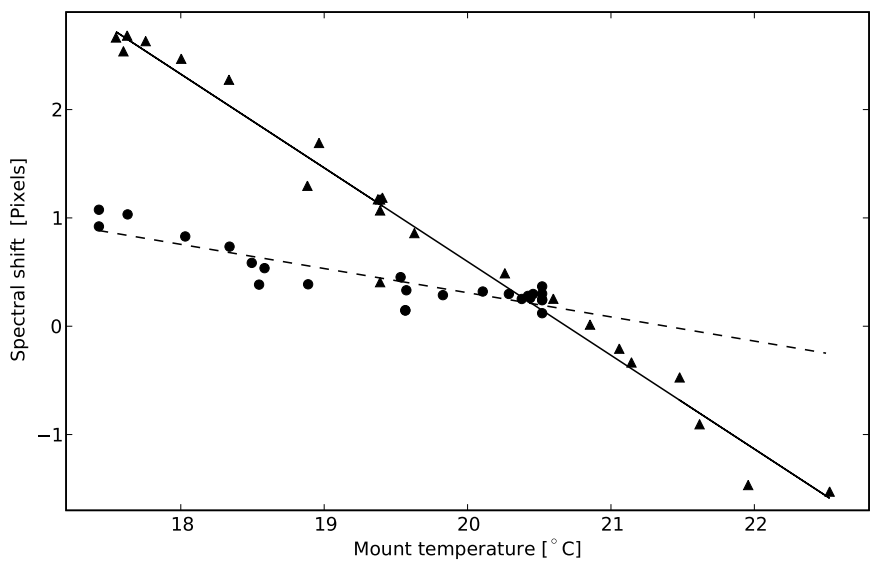

Fig. 8. Measured thermal shift in spectral direction with (dots, dashed line) and without (triangles, solid line) thermal compensators installed in the grating mount.

system keeps the spectrograph air very dry with $\mathrm{RH}_{\mathrm{avg}}=13 \%$ and $\mathrm{RH}_{\max }<30 \%$. The higher outliers correspond with physical interventions in the spectrograph room during commissioning and will disappear in the future.

Temperature. A triple isolation of the spectrograph is the basis of precise thermal control. The spectrograph bench is completely packed by a box of demountable 60 -mm panels of polyurethane sandwiched between thin steel sheets. Inside this box, thermal dissipation is minimal and limited to the photo multiplier of the exposure metre $(\sim 250 \mathrm{~mW})$ and the actuator for the miniature shutter. The latter is a bistable solenoid that only dissipates for a few ms during opening or closing of the shutter. The inner room, built from the same $60-\mathrm{mm}$ isolating panels, surrounds the spectrograph box. A LakeShore Model331 temperature controller with 50-Watt heater actively stabilises the temperature of this room at $18 \pm 0.01{ }^{\circ} \mathrm{C}$. Finally, similar 100 -mm walls for the outer room isolate the spectrograph further from the outside world. An air conditioning system keeps the temperature in this room at $14 \pm 0.1^{\circ} \mathrm{C}$.

Pressure. Apart of temperature, atmospheric pressure changes also have an effect on the refractive index of air, causing a spectral shift. Figure 9 shows typical pressure fluctuations including the (semi-)diurnal atmospheric tide (Lindzen 1979), together with the resulting radial velocity offset that amounts to $0.05 \mathrm{pixel} / \mathrm{hPa}$ or $0.08 \mathrm{~km} /(\mathrm{s} \mathrm{hPa})$.

Housing the spectrograph in a vacuum tank would have been the obvious approach for avoiding pressure-induced instrumental drift. However, practical and financial limitations prohibited this solution. As an alternative, we have started the installation of a system that should stabilise the spectrograph at a constant absolute pressure of $\sim 782 \mathrm{hPa}$, a value close to the maximum atmospheric pressure that occurs at the altitude of the observatory. To allow for over-pressure of maximum $\sim 20 \mathrm{hPa}$ in the spectrograph room during periods of low atmospheric pressure (observing conditions never occur when atmospheric pressure is below $762 \mathrm{hPa}$ ), the walls of the outer room have been reinforced by a structure of $40-\mathrm{mm}$ steel tubes.

\subsection{Optical fibre link}

The large dimensions of HERMES prohibit the installation of the instrument at the telescope focal plane. The obvious solution
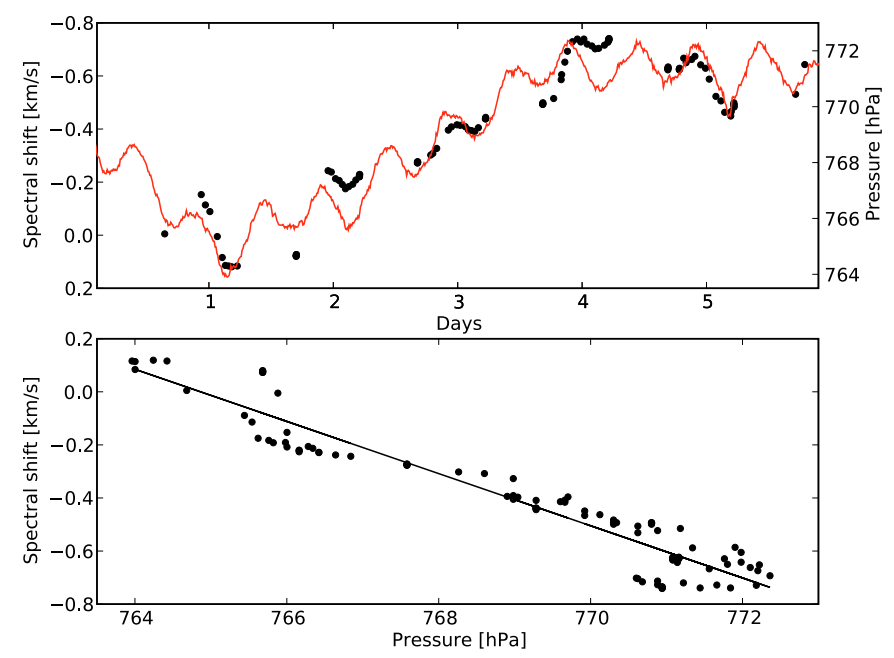

Fig. 9. Radial velocity shift (dots) and typical atmospheric pressure fluctuations (red line) over several days.

is to use optical fibres to relay the light from the telescope to the spectrograph. Aside from causing some light loss, the fibre link offers several clear advantages for instrumental stability:

- The optical fibres provide mechanical isolation between telescope and instrument, completely avoiding gravitational flexure and vibration.

- Mounting the instrument away from the telescope makes it possible to house it in an isolated and precisely controlled chamber, shielding it from all environmental changes in the dome.

- Finally, it is well known that optical fibres tend to scramble the image at the fibre entrance (Barden 1998), reducing the effect of seeing and guiding variations on the illumination of the spectrograph.

Besides the internal absorption, which is small for fibre lengths up to a few dozen metres, the most important source of throughput loss in an optical fibre is caused by focal ratio degradation (FRD). Microbendings, imperfections, and stress in the fibre scatter the propagation angle of the rays of light, resulting in a larger aperture of the output beam or a degraded focal ratio. FRD has a negative effect on the resolution-throughput product of the spectrograph, decreasing at least one of these two parameters. The FRD properties of various fibre types have been examined in different set-ups and several reports about the efficiency of fibres installed on telescopes exist (e.g. Avila 1998; Ramsey 1988). We think it safe to conclude that for most fibres, FRD depends much more on the quality of the fibre polishing, alignment, mounting, glueing, etc. than on the fibre type, so we performed no extensive FRD testing during the selection of the HERMES' fibres. However, fibre confectioning was done with outmost care to keep light loss in the fibre small.

\subsubsection{Optical configuration}

Four optical fibres are used for the HERMES spectrograph: two star fibres mounted in the telescope interface at the focal plane of the Mercator telescope; a calibration fibre between calibration unit and telescope interface for illuminating the star fibres and finally a wavelength reference fibre (WRF) that feeds wavelength calibration light directly from the calibration unit to the spectrograph during observations with simultaneous thorium exposures. All fibres are multi-mode, FBP-type fibres (Polymicro, 


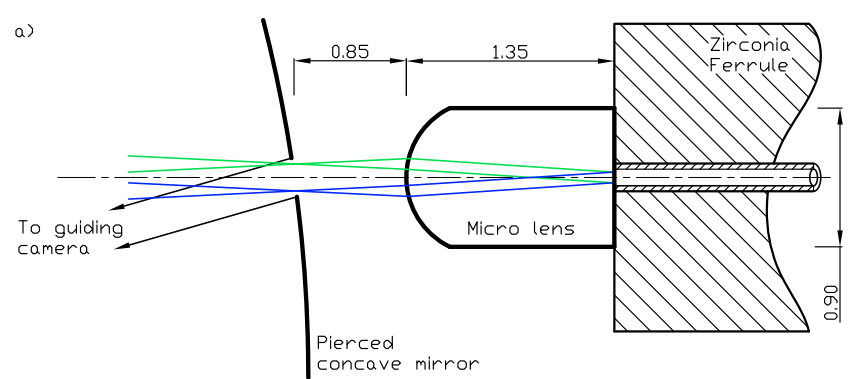

b)

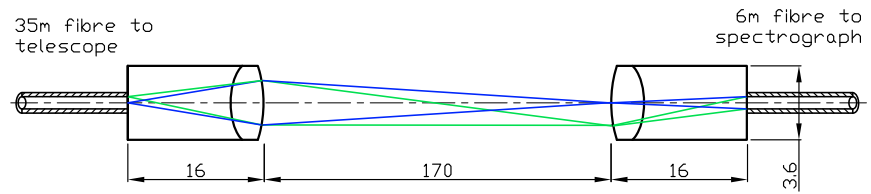

Fig. 10. Optical fibre link: a) fibre entrance (HRF) in telescope focal plane and b) double scrambler with each fibre end imaged on to the other fibre end's pupil. Scaling has been adjusted for clarity, dimensions $(\mathrm{mm})$ have been added for reference.

Table 1. Fibre link characteristics.

\begin{tabular}{lcc}
\hline \hline & LRF & HRF \\
\hline Fibre core diameter & $60 \mu \mathrm{m}$ & $80 \mu \mathrm{m}$ \\
Sky aperture & $2.15 \mathrm{arcsec}$ & $2.50 \mathrm{arcsec}$ \\
Aperture diameter & $150 \mu \mathrm{m}$ & $175 \mu \mathrm{m}$ \\
Microlens: & & \\
$\quad$ Focal length & $0.65 \mathrm{~mm}$ & $0.85 \mathrm{~mm}$ \\
$\quad$ Lens glass type & N-LAK33 & N-BAK1 \\
$\quad$ Glass refractive index $n_{d}$ & 1.75 & 1.57 \\
$\quad$ Radius of curvature & $0.50 \mathrm{~mm}$ & $0.50 \mathrm{~mm}$ \\
$\quad$ Lens thickness & $1.15 \mathrm{~mm}$ & $1.35 \mathrm{~mm}$ \\
$\quad$ Lens diameter & $0.90 \mathrm{~mm}$ & $0.90 \mathrm{~mm}$ \\
Entrance focal ratio & $f / 4.35$ & $f / 4.9$ \\
\hline
\end{tabular}

USA) without important $\mathrm{OH}^{-}$absorption bands in the near IR like many other fibres. The fibre length between telescope and spectrograph is $35 \mathrm{~m}$. The sky fibres are a low-resolution fibre (LRF) with a core diameter of $60 \mu$ m yielding a sky aperture of 2.15 arcsec, and a high-resolution fibre (HRF) with a core diameter of $80 \mu \mathrm{m}$ and a sky aperture of 2.5 arcsec. It might seem contradictory that the larger diameter fibre provides higher resolution, but only this fibre is equipped with a slicer that effectively halves its diameter and doubles spectral resolution. Both fibres have a $125-\mu \mathrm{m}$ diameter cladding so that they can be mounted easily in standard FC ferrules.

An AR-coated plano-convex micro lens (Throl Optics, Germany), glued on top of each star fibre and mounted behind a small aperture in the telescope focal plane, images the telescope pupil onto its rear surface, filling $90 \%$ of the fibre's entrance-surface (Fig. 10a). At the same time, this lens reduces the $f / 12$ telescope focal ratio to $f / 4.9$ for HRF and $f / 4.35$ for LRF. These values are reasonably well-suited to minimising focal-ratio degradation (FRD) in the fibre link, especially since at the other end of the fibres, the spectrograph accepts an aperture of $f / 3.7$. Details of the fibre link optics are summarised in Table 1. It is worth noting that the telescope pupil and not the star image is formed on the fibre entrance. This allows for a much simpler optical and mechanical system.

We estimate the total throughput of the HRF link, including the micro lens coupling, internal absorption, and FRD losses, to be at least $70 \%$. The large sky aperture results in almost negligible seeing losses in case of good (sub-arcsecond) seeing.

\subsubsection{Scrambler}

Although an optical fibre is an almost perfect scrambler of the spatial information at the fibre entrance, even a very long fibre does not completely remove angular information. Since we use pupil imaging, seeing variations, and guiding imprecisions mostly result in angular fluctuations. These may still induce some variation in the illumination of the echelle grating and thus introduce some error in radial-velocity measurements. To further improve illumination stability, we decided to add a double image scrambler as described by Connes (1985), tested by Casse \& Vieira (1997) among others and successfully used on HARPS (Pepe et al. 2000) and others. A symmetric optical system in the middle of the fibre converts the far field of the first half fibre to the near field of the second half and vice versa (Fig. 10b). The pupil of one fibre is imaged onto the entrance of the other fibre, and thus angular non-uniformity is transformed into spatial nonuniformity that is perfectly scrambled by the second part of the fibre. In this way, the residual angular non-uniformity not scrambled by the first part of the fibre link is scrambled by the second. The scrambler consists of two identical doublets at a distance of $170 \mathrm{~mm}$ (Fisba Optik, Switzerland). The exit pupil of the first fibre system fills the $60-\mu \mathrm{m}$ core of the second fibre exactly.

The scrambling gain (SG) is defined as the ratio between the displacement of the star in front of the input fibre end and the shift of the point spread function (PSF) on the spectrograph detector:

$\mathrm{SG}=\frac{d / D}{f / F}$

where $D$ is the fibre diameter, $d$ the star shift, $f$ the shift of the PSF, and $F$ the full width at half maximum of the PSF. We measured a value of 1150 for the near field contribution of the scrambler to the SG. For comparison, the SG of the same $60-\mu \mathrm{m}$ fibre without scrambler was measured to be only 123 .

The improved illumination stability of the grating by the scrambler comes at the cost of lower throughput. The scrambler is therefore only implemented on the low-resolution fibre, optimised for high-stability radial velocity work. The sliced highresolution fibre focuses on high efficiency and does not incorporate a scrambler. We measured the efficiency of the scrambled fibre (LRF) with respect to the HRF to lie around 70\%; however, this is not entirely due to the scrambler alone. The smaller throughput can be partly explained by the smaller LRF fibre diameter (60 versus $80 \mu \mathrm{m})$.

\subsection{Telescope interface and calibration unit}

The telescope interface, installed at the Nasmyth A focal station of the Mercator telescope, links the telescope via the optical fibres to the spectrograph. It includes an atmospheric dispersion corrector (ADC), the fibre entrance, a fibre viewer/telescope guiding system, the calibration light projection optics, and a mask that selectively covers one or both of the star fibres (Fig. 11).

\subsubsection{Atmospheric dispersion corrector}

Although the fibre entrances have a fairly large sky aperture of 2.15 or 2.5 arcsec, HERMES would be limited to observations 


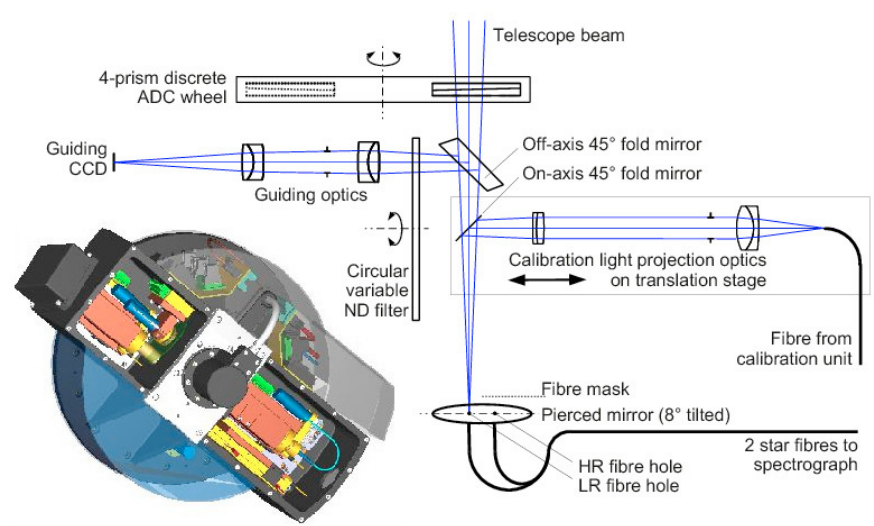

Fig. 11. Telescope interface layout and 3D view.

at zenithal angles smaller than $50^{\circ}$ if we want to avoid losing an important part of the far blue or red flux due to differential atmospheric dispersion. The HERMES atmospheric dispersion corrector provides a step-wise correction of the atmospheric dispersion by means of a set of four correctors in a selection wheel. Each corrector consists of a cemented doublet prism of N-BK7 and LLF1 glass (wedge angles: $0.55^{\circ}, 1.85^{\circ}, 3.60^{\circ}$ and $5.40^{\circ}$ ). The corrector selection matches the requirement that the length of the secondary spectrum never becomes larger than 1 arcsec for zenithal angles up to $65^{\circ}$ and that it stays below 2 arcsec up to $72^{\circ}$.

\subsubsection{Acquisition and guiding}

The fibres, together with their micro lenses, are precisely mounted at the back of a polished stainless steel mirror in the focal plane of the telescope, just in front of the $175-\mu \mathrm{m}$ and $150-\mu \mathrm{m}$ diameter apertures (Fig. 10a). This concave mirror is slightly inclined $\left(8^{\circ}\right)$ to reflect the image of the field via a $45^{\circ}$-fold mirror through the fibre-viewer optics to the guiding $\mathrm{CCD}$. The fibre-viewer optics reduce the telescope $f / 12$ focal ratio to $f / 6.5$, providing a comfortable acquisition field of $4 \times 6$ arcmin on an SBIG ST-1603ME CCD camera $(1020 \times 15309-\mu$ m pixels $)$.

During acquisition, the image of the star is centred on one star-fibre hole, while a movable mask blacks out the other fibre hole. The light of the wings of the PSF that will not enter the star-fibre hole is subsequently used for telescope guiding.

\subsubsection{Calibration}

A separate calibration unit is connected to the telescope interface through a $300-\mu \mathrm{m}$ optical fibre. The calibration light projection optics, mounted on a linear translation stage, can inject calibration light into each of the star fibres separately, thus providing optimal flat-field and wavelength calibration possibilities. The projection optics are designed to deliver a beam with exactly the same focal ratio as the telescope in order to fill the fibres in all cases with a similar light cone.

For wavelength calibration, we use a combination of a thorium-argon lamp equipped with a red-blocking filter to cut off otherwise saturated argon lines, together with a neon lamp for additional lines in the near infrared. A double tungsten lamp is used for good flat-field illumination. This light source consists of a normal low-power lamp providing the red part of the continuum spectrum, combined with a high-power lamp with a red-blocking filter in front of it for the blue part.

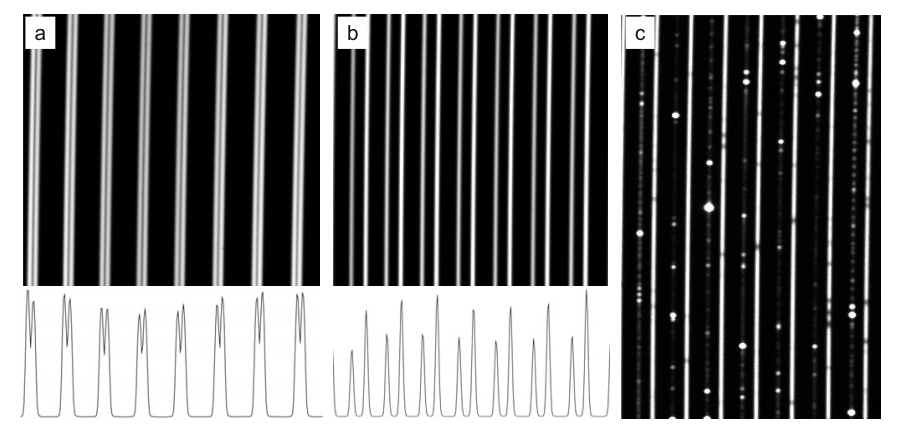

Fig. 12. Small part of raw image for a) HRF flat-field exposure, b) simultaneous LRF and reference fibre flat-field exposure, and c) LRF stellar exposure interlaced with Thorium reference exposure. The crosscuts insets show cross-order profiles of a) the double-peaked sliced HRF and b) LRF and reference fibre (30\% smaller than LRF).

During the simultaneous thorium mode, calibration light is fed directly to the spectrograph through the wavelength reference fibre. A variable neutral density filter in front of this fibre matches the flux from the calibration source to the integration time during this type of exposures.

\subsubsection{Observing modes}

Apart of typical calibration exposures, HERMES basically offers only two science observing modes: a) a high-resolution mode that uses the image slicer and that is optimised for the highest possible throughput and b) a high-precision radial velocity mode using the simultaneous wavelength reference exposure technique. Spectral resolution is reduced in this mode, but at the cost of lower efficiency, spectrograph illumination is more homogenous and stabler because of the double scrambling. As the spectrograph itself has a completely fixed set-up, instrument configuration is done solely through the various mechanisms in the telescope interface and calibration unit. Figure 12 shows a small part of three raw images, illustrating how spectra are recorded on the detector in different observing modes.

HERMES does not offer a sky fibre for background correction. The limited need for sky substraction for high-resolution spectroscopy on a 1.2-m telescope does not justify the increased complexity. The LRF mode will only be used for bright objects, and interleaving a HRF spectrum with a sky spectrum is not possible without additional cross dispersion because of the broad cross-order profile of the sliced fibre. When observing a faint object with full moon, a sky spectrum can always be taken after the science exposure.

\subsection{Detector system}

HERMES uses a standard-silicon, thinned, back-illuminated e $2 \mathrm{v}$ CCD42-90 $(2048 \times 460813.5-\mu$ m pixels $)$ as detector for the spectrograph. After a quantified analysis of spectra taken with a deep-depletion chip, we rejected the use of such a device out of fear of resolution degradation at the bluest wavelengths where the much thicker silicon layer of the deep-depletion device is sensitive to lateral charge diffusion, this despite its higher red efficiency and strongly reduced fringing properties. To solve the fringing problems of the thin $(\sim 15 \mu \mathrm{m})$ standard silicon detector, we adopted a novel solution developed and proposed by e2v Technologies (UK), namely the use of a graded AR coating (Kelt et al. 2006). As the HERMES spectrograph has a completely fixed spectral format, it was possible to optimise locally 


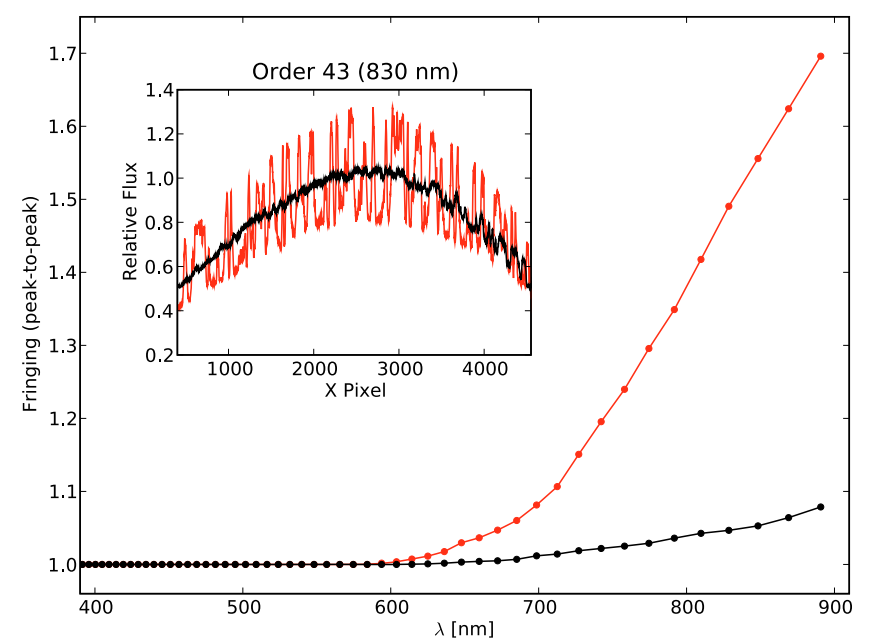

Fig. 13. Peak-to-peak value of fringing, relative to signal flux for a standard (red) and a graded-AR coated CCD (black). The inset shows the flux in the central part of order 43 (around $830 \mathrm{~nm}$ ) with a standard (red) and the graded-AR coated CCD (black), illustrating the different fringing nature of both devices.

the thickness of the AR coating for the wavelengths hitting that region of the detector. The coating thickness follows the spectral gradient in cross-order direction, as well as the curved pattern of the echelle orders. This of course increases the overall detector efficiency, because for each pixel, the AR coating is now matched to the incident monochromatic wavelength. Moreover, fringing at NIR wavelengths is strongly reduced because of the much lower Fresnel reflections at the CCD surface. As can be seen from Fig. 13, the fringing amplitude for the reddest wavelengths of the device with graded AR coating is about 9 times smaller than for a similar device with astronomy broadband AR coating. The reflection gradient of the coating can be seen in the picture of the detector in Fig. 14. The red-sensitive part at the top of the CCD looks blue because red light is efficiently absorbed and detected. As the coating is less efficient for short wavelengths in that part of the chip, mostly blue light is reflected. The picture of a full-frame raw image in Fig. 15 shows the position of the curved echellogram on the CCD.

The detector is mounted in a Infrared Laboratories cryostat and cryogenically cooled by a CryoTiger closed-cycle refrigerating system (Brooks Automation, USA). A LakeShore Model331 temperature controller stabilises the detector temperature at $160 \mathrm{~K}$ with a precision at $0.01 \mathrm{~K}$ level. Detector control and read-out is done through a standard SDSU CCD controller (Astronomical Research Cameras, USA). Reading out all 9 MPixels in slow mode takes $50 \mathrm{~s}$ with a read noise of $5 \mathrm{e}_{\mathrm{rms}}^{-}$ and $30 \mathrm{~s}$ with $8 \mathrm{e}_{\mathrm{rms}}^{-}$in fast mode.

\subsection{Instrument control}

An industrial Linux PC, connected to the various field devices (servo drives, remote $\mathrm{I} / \mathrm{O}$ and variable power supplies) by RS-232 and RS-485 point-to-point and bus networks, controls the HERMES instrument. Drivers have been written in Python to extend the Mercator Observatory Control System (MOCS), currently under development (Pessemier et al. 2010). This software is component-based, which means that HERMES could easily be integrated within the global control system by adding components for the detector, the instrument control, and the associated graphical user interfaces (GUI). The instrument control software

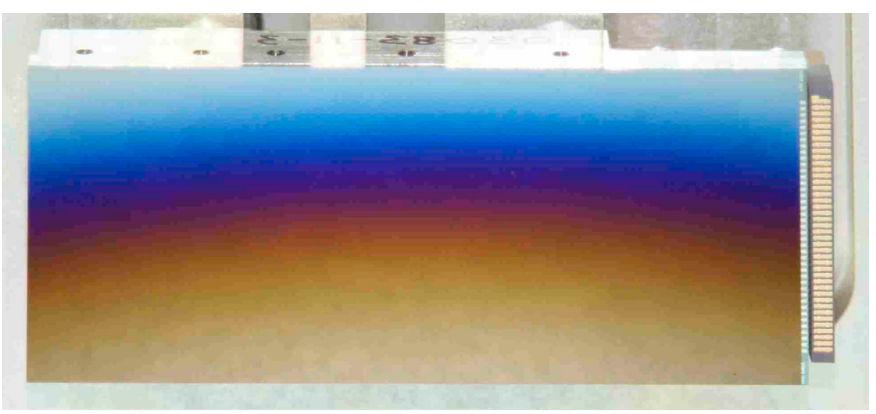

Fig. 14. Picture of the graded-AR coated CCD. The red-sensitive part at the top of the chip looks blue because red light is absorbed while blue light is reflected.

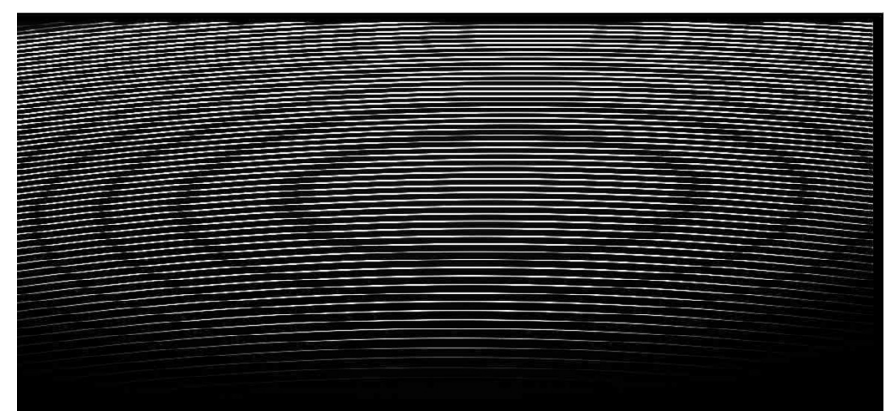

Fig. 15. Full-frame raw image of a flat-field spectrum in the lowresolution fibre.

acts as a bus master for the industrial hardware and implements the low and high level tasks in an object-oriented way with multiple layers of abstraction. All relevant data is published to the network, and any interested party (such as the GUIs) can subscribe to it. Integrating HERMES also required extending both the auto-guiding system (to allow target acquisition and centroiding on a fibre image) and the scheduling software. A GUI is available for the queue scheduling of complete nights of observations, thereby specifying the instrument settings and exposure parameters for each target. The observer has to supervise the system and intervene whenever needed, while the MOCS software handles the queued observations fully automatically.

\section{Integrated data reduction pipeline}

The data reduction pipeline performs the traditional corrections for the bias level, the inter-order background level, the fringing on the detector, and the modulation of the intensity in each spectral order (blaze function) and applies a pre-normalisation eliminating the global wavelength-dependency of the flat-field calibration system. It determines in a robust way the dependency of the positions of the spectral orders on time-dependent factors, extracts the flux in each order with the options of estimating the total flux in a cross-cut from the pixels free of cosmic rays and of weighing pixels using a cross-order flux distribution model. Final spectra can be represented order per order in tabular form, assigning a wavelength to each pixel, or resampled to bins with a size of $\approx 1.6 \mathrm{~km} \mathrm{~s}^{-1}$ (the natural pixel size on average over each order) or with a fixed wavelength step, either over the whole wavelength region, over part of it, or just the "natural" step over each order. Depending on the choice, spectra may be merged over several or all orders. Care is taken not to include parts of orders far out of the free spectral range where the risk is high that systematic bias dominates random noise. A greatly simplified data reduction option is available for fast first-look purposes. 

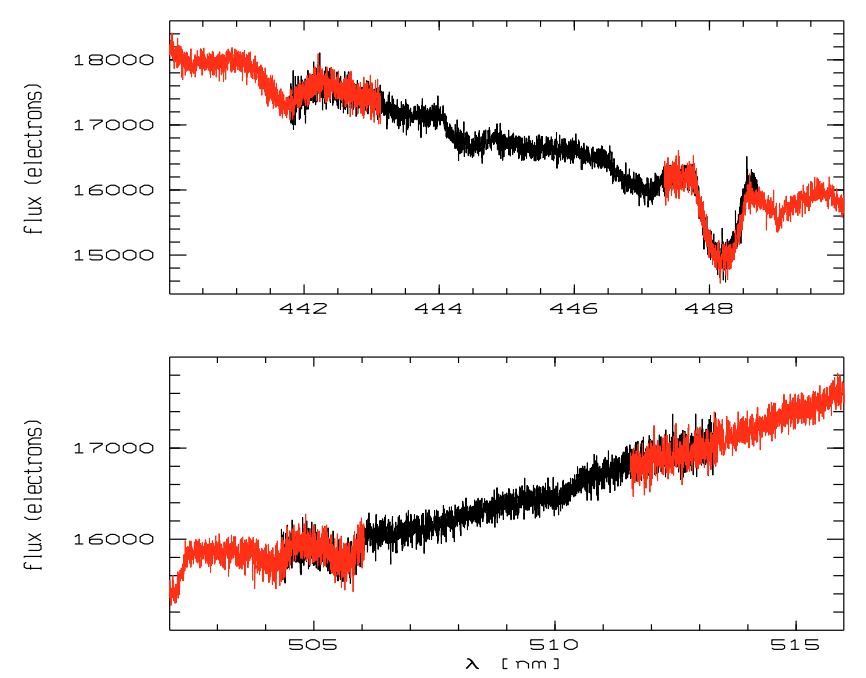

Fig. 16. Selected regions of a late-B type binary, each panel showing a spectral order in black and the two neighbouring orders in red.

Presently, the data reduction pipeline works in a frame-perframe mode, but the final goal is to execute a number of reduction steps in a differential mode to gain robustness in the temporal model of the spatial and wavelength geometry on the detector (Hensberge 2007). A detailed description of the data reduction procedures will be presented in a forthcoming paper. For the moment, we draw attention to the excellent agreement in the intensity level of the extracted spectra over the common wavelengths in subsequent orders. Figure 16 shows selected regions of the spectrum of a late-B type binary. For each order, the used wavelength range extends approximately over the range where the intensity of the blaze is higher than one third of its value at maximum. The extraction is excellent and allows for detailed full spectral reconstruction, even for broad shallow lines at the edges of spectral orders.

All raw and reduced frames are archived. A web-based interface for accessing the HERMES database is under development. The archive also provides input for the scheduler software when composing the observing queues.

\section{Performances}

\subsection{Spectral resolution}

Compared to many other spectrographs and despite a comfortably large sky aperture, HERMES really is a high-resolution spectrograph. In Fig. 17 (bottom panel) spectral resolution is shown as derived from the measured line widths on a spectrum of thorium, argon, and neon emission lines. In LRF mode, spectral resolving power $R=\lambda / \Delta \lambda$ amounts to $63000\left(4.8 \mathrm{~km} \mathrm{~s}^{-1}\right)$. This value is $23 \%$ higher than the value of 51000 , calculated from a ZEMAX model for a 2.15-arcsec rectangular slit. In HRF mode we measure $R=85000\left(3.5 \mathrm{~km} \mathrm{~s}^{-1}\right), 12 \%$ higher than the calculated value of 76000 , proving the excellent image quality of the spectrograph optics.

The anamorphic magnification of the spectrograph makes the sampling drop along the spectral orders (Fig. 17, top panel). For HRF, sampling decreases from 2.7 to 2 pixels per resolution element, and this explains the drop in HRF resolution with $\mathrm{X}$-pixel coordinate. Only for the lowest sampling values does the image quality of the optics start becoming relevant, causing a small loss in resolution. This effect is not noticeable for LRF because of much higher sampling (3.8-3.1 pixels). Figure 18

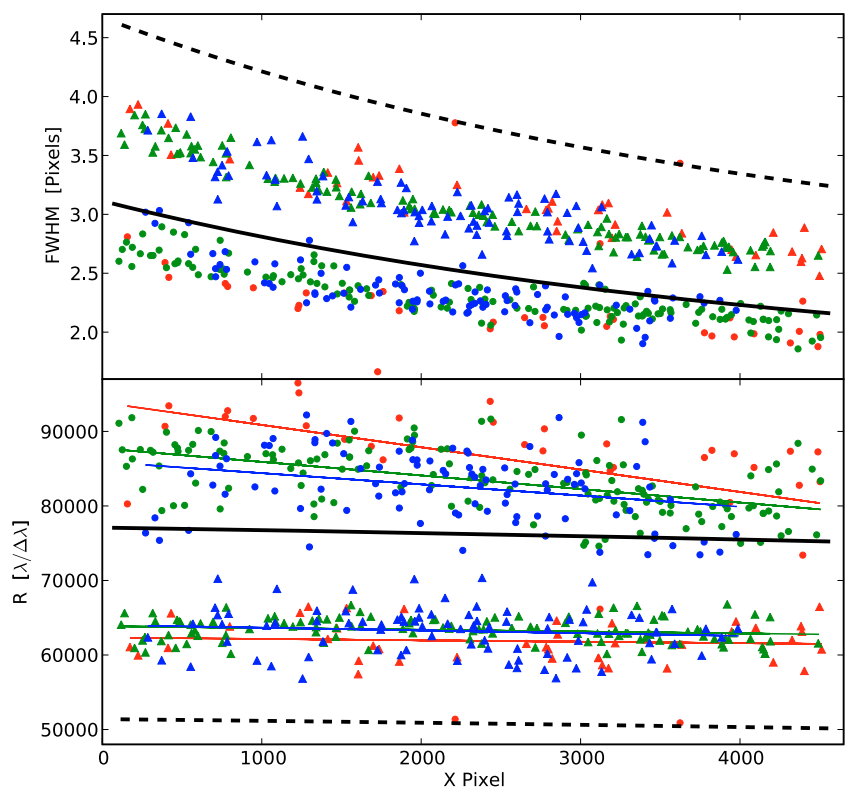

Fig. 17. Measured line width (top panel) and spectral resolution (bottom panel) for HRF (dots) and LRF (triangles) along the detector columns. Colours correspond to wavelength for three groups of spectral orders. For comparison, the solid and dashed thick black lines show the width and resolution calculated in ZEMAX for HRF and LRF respectively.

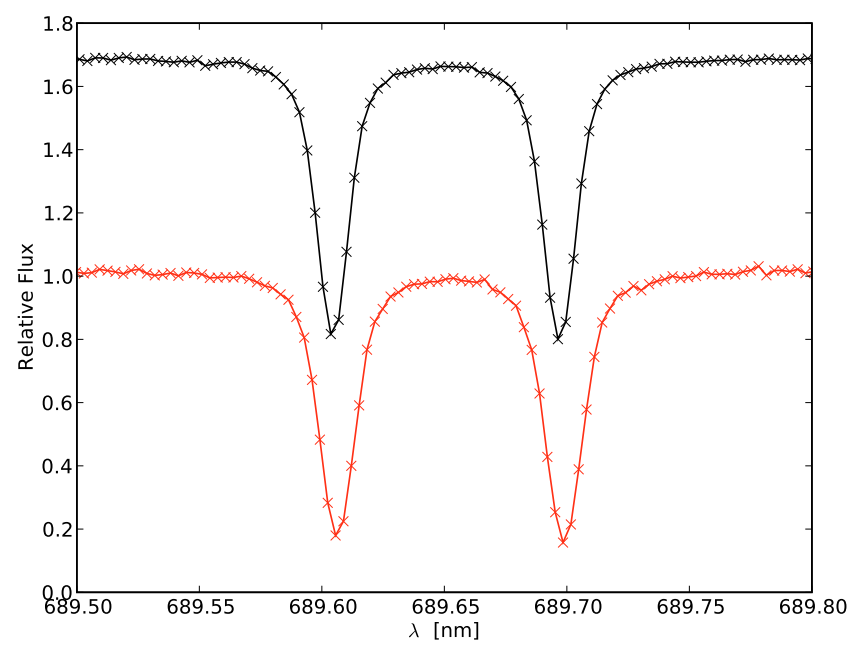

Fig. 18. Part of normalised spectrum with two $\mathrm{O}_{2}$ lines measured in LRF (red) and, shown with an offset, in HRF (black).

shows the accurate sampling in a detailed part of the spectrum with two $\mathrm{O}_{2}$ lines, both for HRF and LRF. Figure 19 shows a larger part of two more illustrative spectra (Procyon and Polaris).

\subsection{Efficiency}

Figure 20 shows the efficiency at blaze peak for measurements under excellent observing conditions. Unfortunately, according to the histogram in Fig. 4 median observations only reach $60 \%$ of these values. The complete system including spectrograph and telescope has a maximum throughput of $17 \%$ for $\mathrm{HRF}$ and $12 \%$ for LRF around $500 \mathrm{~nm}$. Taking out telescope losses (estimated to be $40 \%$ for three reflections), these numbers leave us with a peak efficiency for the spectrograph alone of around $28 \%$ and $20 \%$, outperforming many other first-class instruments. 


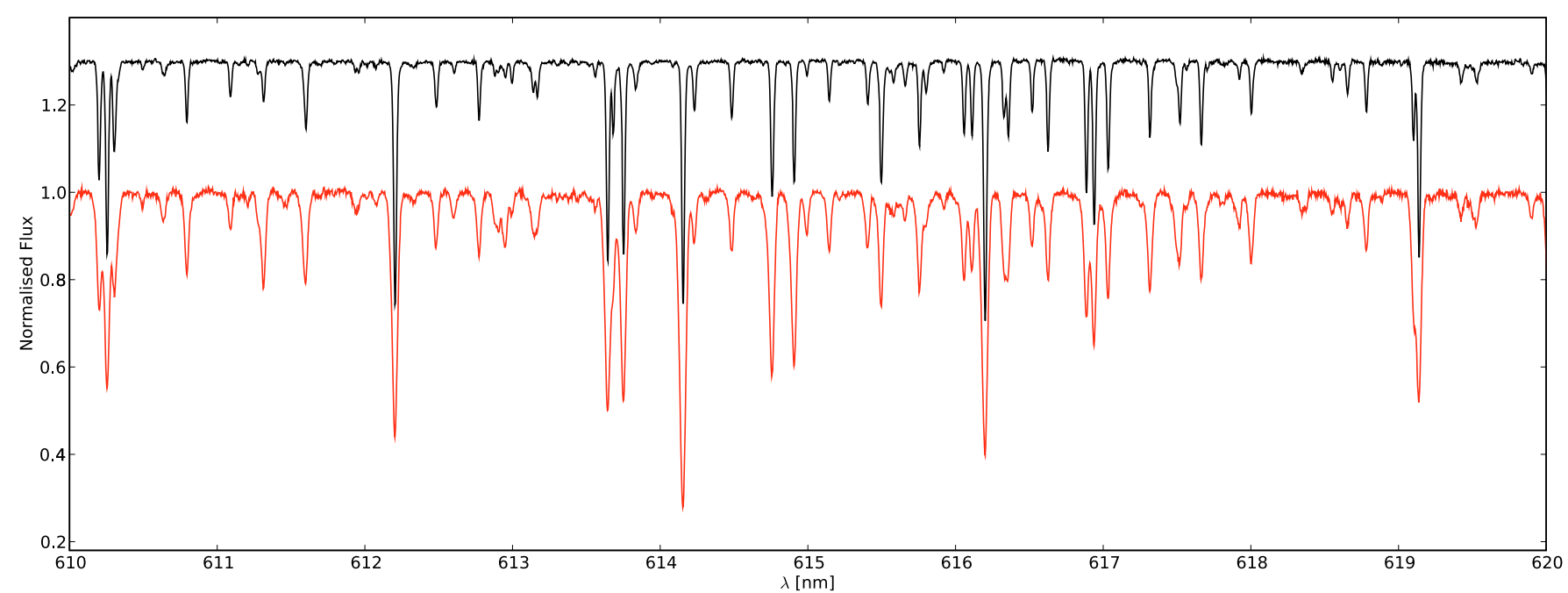

Fig. 19. Illustrative spectra of Procyon (F5IV, black) and Polaris (F7:Ib-IIv, red). Only a small part of the spectral range is shown.

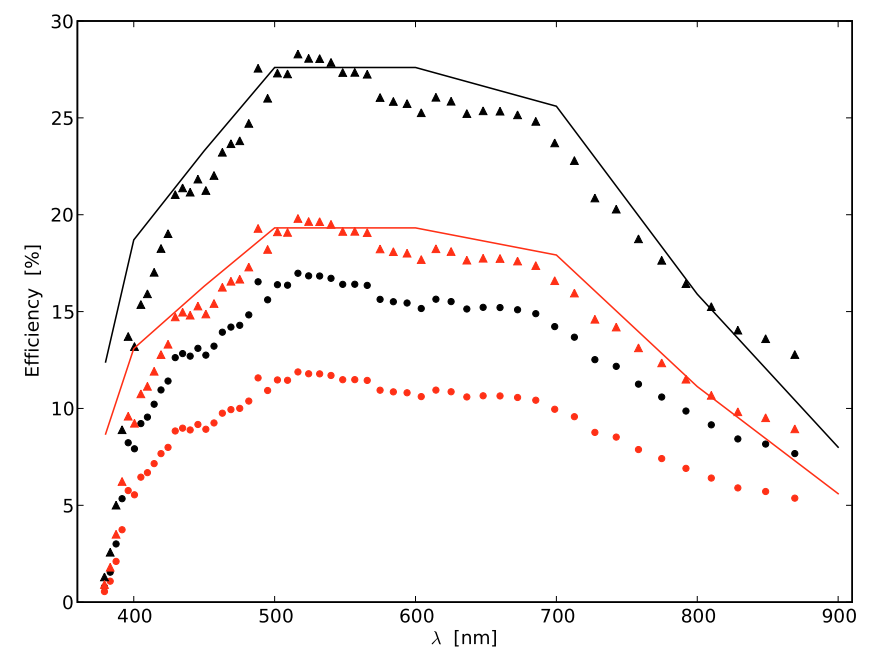

Fig. 20. Measured total efficiency of HERMES + Mercator telescope (dots) and efficiency of spectrograph only, including fibres and detector (triangles) for HRF (black) and LRF (red). The solid lines show the calculated spectrograph efficiency.

Apart from the bluest wavelengths where HERMES performs below expectations, the measured throughput corresponds quite well to calculated values based on measurements, data sheets, and specifications and where necessary, founded estimations of the throughput of the individual components including fibres, mirrors, dispersers, lenses, and CCD.

With this efficiency and during good quality nights, a one hour HRF exposure on a star of $m_{V}=10.4$ yields an $S / N$ of 100 per pixel around $550 \mathrm{~nm}$, decreasing to $S / N=20$ with $m_{V}=13.4$. For LRF exposures, we obtain a similar $S / N$ with stars of $m_{V}=10.0$ and 13.2.

\subsection{Stability}

The first year of operation was almost entirely devoted to science exploitation using the HRF mode. The spectrograph and pipeline performances are therefore best tested for this mode of operation. Furthermore, the pipeline developments for the LRF mode have not yet been completely finalised.

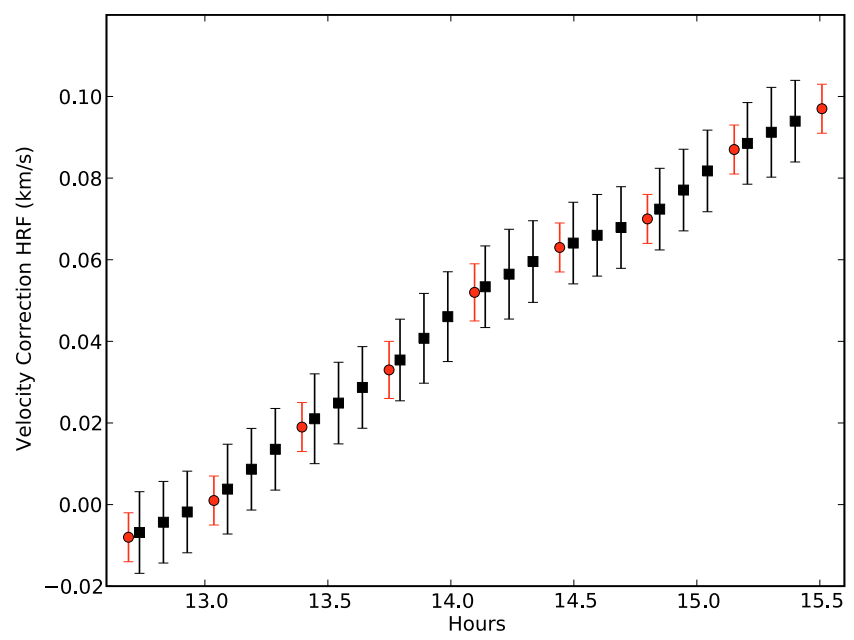

Fig. 21. Continous monitoring the instrumental drift of HERMES in HRF mode (red circles) and the interpolation during mid-exposures of the science target (black squares).

During a night, the short-term stability of the spectrograph is strongly affected by the atmospheric pressure variations (Sect. 2.1.3). To quantify the real velocity stability of HERMES in HRF mode, we interleaved independent spectra of the radial velocity standard HD 164922 with wavelength calibration frames. To correct for these short-term instrumental drifts, we corrected the radial velocities obtained for HD 164922 by interpolating between the shifts found on the basis of the neighbouring calibration frames (see Fig. 21). A standard deviation of $2.9 \mathrm{~m} \mathrm{~s}^{-1}$ is found on 24 datapoints. After sigma clipping of $2 \sigma$, this reduces to $2.3 \mathrm{~m} \mathrm{~s}^{-1}$. Despite the lower resolution of the LRF fibre, but since it is equipped with an optical scrambler, and as the reference fibre allows for simultaneous correction of the instrumental drift, this mode offers a slightly higher accuracy with the standard deviation reduced to $2.5 \mathrm{~m} \mathrm{~s}^{-1}$ on 45 datapoints and to $2.0 \mathrm{~m} \mathrm{~s}^{-1}$ after $2 \sigma$ clipping. The drawback is the less-efficient throughput so that this mode should be reserved for bright objects only where the errors in velocity space are dominated by drift correction and not by photon noise, or for 


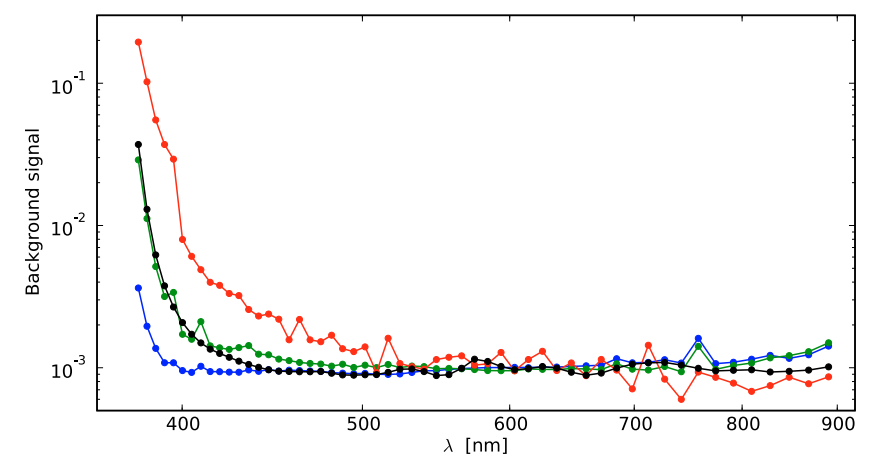

Fig. 22. Ratio of signal level in the inter-order region to the flux in the adjacent orders for a flat field spectrum (black) and for an O, F, and M star (blue, green, and red curves).

observations where sampling requirements prohibit the sequential interleaving of wavelength calibration exposures.

Although the cold head of the CryoTiger cooler does not contain any moving parts, possible vibrations caused by the gas expansion or transmitted from the compressor through the flexible gas lines (15 m long), still raise some concern. We tried to measure these vibrations and found that, fortunately, in an experiment consisting of 300 measurements with a total time base of $4.5 \mathrm{~h}$, they are not noticeable at the detection limit of $\sigma=70 \mathrm{~cm} \mathrm{~s}^{-1}$. The same experiment showed that also the operation of a turbo-molecur vacuum pump, connected to the detector cryostat through two metres of flexible vacuum pipe, did not cause any detectable vibrations.

The long-term stability during the first year of operation is compromised by the continuous adjustment and optimisation of the acclimatisation control system. In our normal mode of operation, we use only wavelength calibration frames obtained in the evening and morning of an observation night to calibrate a given science frame in wavelength. Nightly we obtain spectra of IAU velocity standards. Despite the variable environmental conditions, but using the pressure calibration of Sect. 2.1.3, a standard deviation of $50 \mathrm{~m} \mathrm{~s}^{-1}$ is found using all 50 exposures of the IAU standard HD 164922 in between 2009-07-09 and 2010-06-28.

\subsection{Scattered light and ghosts}

Scattered light is usually an important issue in an echelle spectrograph. Grating efficiency is typically $60-70 \%$, and a large part of the lost flux ends up scattered throughout the instrument. Nevertheless, careful design that includes efficient baffling and use of high-quality optical components, allow HERMES to deliver very clean spectra, comparing favourably with many other echelle spectrographs. As can be appreciated from Fig. 22, the distribution of scattered light is very local. The inter-order background signal fluctuates around $0.1 \%$ of the flux in the adjacent orders. Only when the signal level is very low (particularly at short wavelengths) does a global straylight component become relevant.

HERMES spectra are virtually free of ghost images. In spectra of the emission line star P Cygni with, amongst others, a strongly saturated $\mathrm{H} \alpha$ line, it turned out to be impossible to detect the presence of ghost images of the emission lines.

\section{Conclusion}

We presented in this contribution the design, manufacturing, and performance analyses of HERMES, a high-resolution fibre-fed
Table 2. HERMES main parameters.

\begin{tabular}{|c|c|}
\hline \multicolumn{2}{|l|}{ Spectral resolution } \\
\hline HRF & 85000 (with slicer) \\
\hline LRF & $\begin{array}{c}63000 \text { (interlaced with simultaneous } \\
\text { Thorium spectrum) }\end{array}$ \\
\hline \multicolumn{2}{|l|}{ Fibre aperture } \\
\hline HRF & $2.5 \operatorname{arcsec}(80-\mu \mathrm{m}$ fibre $)$ \\
\hline LRF & $2.15 \operatorname{arcsec}(60-\mu \mathrm{m}$ fibre $)$ \\
\hline \multicolumn{2}{|l|}{ Sampling } \\
\hline HRF & $2-2.7$ pixels \\
\hline LRF & $3.1-3.8$ pixels \\
\hline Spectral range & $377-900 \mathrm{~nm}$ (in 55 orders) \\
\hline Spectrograph layout & White-pupil layout on $1.2 \times 2.4 \mathrm{~m}$ bench \\
\hline Beam diameter & $152 \mathrm{~mm}$ \\
\hline Collimator focal length & $1400 \mathrm{~mm}(f / 9.2)$ \\
\hline Echelle grating & $\theta=69.74^{\circ}, 52.676 \mathrm{gr} / \mathrm{mm}, 154 \times 408 \mathrm{~mm}$ \\
\hline Cross disperser & 2 PBL1Y prisms, $37.4^{\circ}$ apex angle \\
\hline Camera focal length & $475 \mathrm{~mm}(f / 3.1,6$ lenses $)$ \\
\hline Detector & $\begin{array}{l}2048 \times 460813.5-\mu \mathrm{m} \text { pixels, thinned BI } \\
\text { CCD with graded AR coating }\end{array}$ \\
\hline \multicolumn{2}{|l|}{ Peak efficiency } \\
\hline HRF & B: $21 \%, \mathrm{~V}: 28 \%, \mathrm{R}: 25 \%, \mathrm{I}: 17 \%$ \\
\hline LRF & B: $15 \%, \mathrm{~V}: 20 \%, \mathrm{R}: 18 \%, \mathrm{I}: 12 \%$ \\
\hline Limiting magnitudes & $1 \mathrm{~h}$ exposure \\
\hline$S / N_{V}=20$ & $m_{V}=13.4(\mathrm{HRF}), m_{V}=13.2(\mathrm{LRF})$ \\
\hline$S / N_{V}=100$ & $m_{V}=10.4(\mathrm{HRF}), m_{V}=10.0(\mathrm{LRF})$ \\
\hline Radial velocity error & $\sim 2.5 \mathrm{~m} \mathrm{~s}^{-1}$ (short term) \\
\hline
\end{tabular}

spectrograph project. We showed that the instrument was built according to the demanding requirements, which resulted in an instrument of excellent stability and unsurpassed efficiency. The main characteristics of our instrument are given in Table 2. The ZEMAX description of the optimised optical system can be found in Appendix A.

After commissioning on the Mercator telescope in April 2009, HERMES immediately became the work-horse instrument. During the first year of operations, this instrument obtained about 7300 spectra on the sky, which makes clear that HERMES also fulfills its robust operational requirements. HERMES is open to the community at large through collaboration with the consortium members and through the Spanish CAT time allocation programme, available for all telescopes at the Roque de los Muchachos observatory.

Acknowledgements. The HERMES project and team acknowledge support from the Fund for Scientific Research of Flanders (FWO) under the grant G.0472.04, from the Research Council of K.U. Leuven under grant GST-B4443, from the Fonds National de la Recherche Scientifique under contracts IISN 4.4506.05 and FRFC 2.4533.09, and financial support from Lotto (2004) assigned to the Royal Observatory of Belgium to contribute to the hardware of the spectrograph. We would like to express our appreciation and gratitude to Francesco Pepe (Geneva Observatory, Switzerland), Otmar Stahl (Heidelberg, Germany), and Ramon García Lopez (IAC, Spain), who served as experts in the review committee, and to José Luis Rasilla (IAC, Spain) for his helpful comments on the optical design.

\section{Appendix A: ZEMAX optical description}

Table A.1 summarises the as built ZEMAX data of all optical surfaces of the HERMES spectrograph. The optical system has been optimised by considering the measured values of the refractive indices of the optical glasses. This means that the use of standard refractive index values results in a slightly suboptimal image quality for the system that is described here. 
Table A.1. ZEMAX optical surface data.

\begin{tabular}{|c|c|c|c|c|c|c|c|}
\hline Surface & Type & Radius & Thickness & Glass & Diameter & Conic & Comment \\
\hline OBJECT & STANDARD & Infinity & 0 & SILICA & 0.62 & 0 & FIBRE EXIT \\
\hline 1 & STANDARD & Infinity & 12 & SILICA & 0.62 & 0 & FN DOUBLET \\
\hline 2 & STANDARD & 3.157 & 3.5 & S-FPL53 & 5.6 & 0 & \\
\hline 3 & STANDARD & -6.048 & 24 & & 5.6 & 0 & \\
\hline STOP & STANDARD & Infinity & 39.5 & & 4.6 & 0 & \\
\hline 5 & STANDARD & 28.04 & 3.05 & S-BAL12 & 12 & 0 & FN TRIPLET \\
\hline 6 & STANDARD & 14.009 & 5.83 & S-FPL53 & 12 & 0 & \\
\hline 7 & STANDARD & -14.009 & 3.06 & S-BAL12 & 12 & 0 & \\
\hline 8 & STANDARD & -28.04 & 59.51 & & 9.1 & 0 & \\
\hline 9 & STANDARD & Infinity & 12.5 & SILICA & 25 & 0 & SLICER \\
\hline 10 & STANDARD & Infinity & 12.5 & SILICA & 25 & 0 & SLICER \\
\hline 11 & STANDARD & Infinity & 1401.48 & & 2.424 & 0 & \\
\hline 12 & COORDBRK $^{a}$ & - & 0 & & - & - & OFF-AXIS ANGLE \\
\hline 13 & STANDARD $^{b}$ & -2802.96 & -1401.48 & MIRROR & 660 & -1 & MAIN COLLIMATOR \\
\hline 14 & COORDBRK $^{c}$ & - & 0 & & - & - & ECHELLE TILT \\
\hline 15 & COORDBRK $^{d}$ & - & 0 & & - & - & LITTROW ANGLE \\
\hline 16 & DGRATING $^{e}$ & Infinity & 0 & MIRROR & 444.71 & 0 & ECHELLE GRATING \\
\hline 17 & COORDBRK $^{f}$ & - & 0 & & - & - & \\
\hline 18 & COORDBRK $^{g}$ & - & 1401.48 & & - & - & \\
\hline 19 & STANDARD $^{h}$ & -2802.96 & -1300 & MIRROR & 660 & -1 & MAIN COLLIMATOR \\
\hline 20 & STANDARD & Infinity & 1504.11 & MIRROR & 114.80 & 0 & FLAT FOLD MIRROR \\
\hline 21 & STANDARD $^{i}$ & -2802.96 & -1280 & MIRROR & 660 & -1 & TRANSFER COLLIMATOR \\
\hline 22 & COORDBRK $^{j}$ & - & 0 & & - & - & PRISM ANGLE OF INCIDENCE \\
\hline 23 & STANDARD & Infinity & 0 & PBL1Y & 196 & 0 & CD PRISM1 \\
\hline 24 & COORDBRK $^{k}$ & - & -71 & & - & - & PRISM HALF APEX ANGLE \\
\hline 25 & COORDBRK $^{l}$ & - & 0 & & - & - & PRISM HALF APEX ANGLE \\
\hline 26 & STANDARD & Infinity & 0 & & 196 & 0 & \\
\hline 27 & COORDBRK $^{l}$ & - & -106 & & - & - & PRISM ANGLE OF INCIDENCE \\
\hline 28 & COORDBRK $^{l}$ & - & 0 & & - & - & PRISM ANGLE OF INCIDENCE \\
\hline 29 & STANDARD & Infinity & 0 & PBL1Y & 196 & 0 & CD PRISM2 \\
\hline 30 & COORDBRK $^{k}$ & - & -71 & & - & - & PRISM HALF APEX ANGLE \\
\hline 31 & COORDBRK $^{k}$ & - & 0 & & - & - & PRISM HALF APEX ANGLE \\
\hline 32 & STANDARD & Infinity & 0 & & 196 & 0 & \\
\hline 33 & COORDBRK $^{l}$ & - & -75 & & - & - & PRISM ANGLE OF INCIDENCE \\
\hline 34 & STANDARD & -316.45 & -21.19 & S-FPL53 & 172 & 0 & CAMERA LENS 1 \\
\hline 35 & STANDARD & 1216.54 & -1.053 & & 172 & 0 & \\
\hline 36 & STANDARD & -331.92 & -16.127 & S-BAL42 & 172 & 0 & LENS2 \\
\hline 37 & STANDARD & -165.26 & -35.949 & S-FPL53 & 164 & 0 & LENS3 \\
\hline 38 & STANDARD & 437.42 & -15.105 & S-LAL12 & 164 & 0 & LENS4 \\
\hline 39 & STANDARD & -580.77 & -311.064 & & 164 & 0 & \\
\hline 40 & STANDARD & -326.08 & -21.277 & PBL25Y & 154 & 0 & LENS5 \\
\hline 41 & STANDARD & 1700.88 & -199.41 & & 154 & 0 & ADJUST FOCUS \\
\hline 42 & STANDARD & 133.26 & -14.1 & SILICA & 88 & 0 & FIELD LENS \\
\hline 43 & TOROIDAL & 224 & -5.3 & VACUUM & 80 & 0 & CYLINDRICAL SURFACE \\
\hline IMAGE & STANDARD & Infinity & & & 41 & 0 & $\mathrm{CCD}$ \\
\hline
\end{tabular}

Notes. ${ }^{(a)}$ Tilt about Y: 6.32; ${ }^{(b)}$ Decenter X before surface: 155, Decenter X after surface: -155 ; ${ }^{(c)}$ Tilt about Y: 0.8; ${ }^{(d)}$ Tilt about X: 69.74; ${ }^{(e)}$ Lines/ $\mu \mathrm{m}$ : 0.052676, Diffraction order: 40-94; ${ }^{(f)}$ Tilt about X: $-69.74 ;{ }^{(g)}$ Tilt about Y: $-0.8 ;{ }^{(h)}$ Decenter X before surface: 155 ; ${ }^{(i)}$ Decenter X after surface: $158 ;{ }^{(j)}$ Tilt about Y: $-29.545 ;{ }^{(k)}$ Tilt about Y: $18.7 ;^{\left({ }^{(l)}\right.}$ Tilt about Y: -29.545.

\section{References}

Aerts, C., Christensen-Dalsgaard, J., \& Kurtz, D. W. 2010, Asteroseismology (Springer Verlag)

Avila, G. 1998, in Fiber Optics in Astronomy III, ed. S. Arribas, E. Mediavilla, \& F. Watson, ASP Conf. Ser., 152, 44

Baranne, A. 1988, in ESO Conference on Very Large Telescopes and their Instrumentation, 2, 1195

Baranne, A., Queloz, D., Mayor, M., et al. 1996, A\&AS, 119, 373

Barden, S. C. 1998, in Fiber Optics in Astronomy III, ed. S. Arribas, E. Mediavilla, \& F. Watson, ASP Conf. Ser., 152, 14

Blomme, R. 2010, High Energy Phenomena in Massive Stars ASP Conf. Ser., ed. J. Marti, P. L. Luque-Escamilla, \& J. A. Combi (San Francisco: ASP), 422,178

Casse, M., \& Vieira, F. 1997, in Society of Photo-Optical Instrumentation Engineers (SPIE) Conf. Ser., ed. A. L. Ardeberg, 2871, 1187

Connes, P. 1985, Ap\&SS, 110, 211

Dekker, H., D’Odorico, S., Kaufer, A., Delabre, B., \& Kotzlowski, H. 2000, in SPIE Conf. Ser. 4008, ed. M. Iye, \& A. F. Moorwood, 534
Hensberge, H. 2007, in The Future of Photometric, Spectrophotometric and Polarimetric Standardization, ed. C. Sterken, ASP Conf. Ser., 364, 275 Hensberge, H., Ilijić, S., \& Torres, K. B. V. 2008, A\&A, 482, 1031

Jorissen, A., Frankowski, A., Famaey, B., \& van Eck, S. 2009, A\&A, 498, 489 Kaufer, A. 1998, in Fiber Optics in Astronomy III, ed. S. Arribas, E. Mediavilla, \& F. Watson, ASP Conf. Ser., 152, 337

Kaufer, A., \& Pasquini, L. 1998, in SPIE Conf. Ser. 3355, ed. S. D’Odorico, 844 Kelt, A., Harris, A., Jorden, P., \& Tulloch, S. 2006, in Scientific Detectors for Astronomy 2005, ed. J. E. Beletic, J. W. Beletic, \& P. Amico, 369

Lehmann, H., Vitrichenko, E., Bychkov, V., Bychkova, L., \& Klochkova, V. 2010, A\&A, 514, A34

Lindzen, R. S. 1979, Ann. Rev. Earth Planet. Sci., 7, 199

Masseron, T., Johnson, J. A., Plez, B., et al. 2010, A\&A, 509, A93

Pepe, F., Mayor, M., Delabre, B., et al. 2000, in SPIE Conf. Ser. 4008, ed. M. Iye, \& A. F. Moorwood, 582

Pessemier, W., Raskin, G., Prins, S., et al. 2010, in SPIE Conf. Ser., 7740

Ramsey, L. W. 1988, in Fiber Optics in Astronomy, ed. S. C. Barden, ASP Conf. Ser., 3, 26

Reyniers, M., Abia, C., Van Winckel, H., et al. 2007, A\&A, 461, 641

Van Winckel, H., Lloyd Evans, T., Briquet, M., et al. 2009, A\&A, 505, 1221 